\title{
EL IMPACTO DEL COSTO POR PROCESOS Y LA CADENA PRODUCTIVA DEL ESPÁRRAGO EN LA AGROEXPORTACIÓN
}

\section{THE IMPACT OF THE COST BY PROCESSES AND THE PRODUCTIVE CHAIN OF ASPARAGUS IN AGROEXPORTATION}

\author{
Nelly Adriana Risco Mc Gregor ${ }^{*}$ \\ ariscomcgregor@gmail.com \\ ${ }^{1}$ Universidad Nacional Mayor de San Marcos, Lima, Perú
}

*Correspondencia: Nelly Adriana Risco Mc Gregor. Email: ariscomcgregor@gmail.com

Recibido: 11.08.21 | Aprobado: 20.08.21

\section{RESUMEN}

Objetivo: Demostrar el impacto positivo del sistema de costos por procesos en la cadena productiva del espárrago en las empresas agroexportadoras, esto dada la necesidad de conocer, de manera confiable, sus costos y que esto, a su vez, les permita mejorar la toma de decisiones. Método: La investigación fue de tipo descriptiva y el diseño de investigación no experimental. Para nuestro análisis, el estudio de caso se aplicó a la empresa Vivadis Perú S.A.C., empresa dedicada a la agroexportación de espárragos. Resultados: El sistema de costos por procesos, aplicado a la cadena productiva del espárrago, permitió determinar de manera más precisa los costos que se manejan por materiales y mano de obra en cada una de las etapas del proceso productivo del espárrago frente a un sistema de costeo manejado de forma empírica cuyos datos se muestran muy generales. Esta actividad contable permitirá proyectar la inversión en la agroexportación. Conclusión: El sistema de costos por procesos impacta favorablemente en la cadena productiva del espárrago, tanto en la aplicación de la materia prima como en la mano de obra utilizada en cada proceso de la cadena productiva.

Palabras clave: espárrago, etapas, producción, costos por proceso.

\section{ABSTRACT}

Objective: To demonstrate the positive impact of the cost system for processes in the productive chain of asparagus in agro-export companies, this given the need to know, in a reliable way, their costs and that this, in turn, allows them to improve the taking of decisions. Method: The research was descriptive and the research design was nonexperimental. For our analysis, the case study was applied to the company Vivadis Perú S.A.C., a company dedicated to the agro-export of asparagus. Results: The process costs system, applied to the asparagus production chain, allowed to determine more precisely the costs that are managed by materials and labor in each of the stages of the asparagus production process compared to a system of costing managed in an empirical way whose data are very general. This accounting activity will make it possible to project investment in agro-exports. Conclusion: The process cost system has a favorable impact on the asparagus production chain, both in the application of raw material and in the labor used in each process of the production chain.

Keyword: asparagus, stages, production, costs per process.

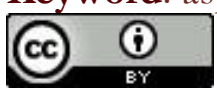




\section{INTRODUCCIÓN}

En el contexto nacional, la actividad agropecuaria es una de las de mayor relevancia económica para el país. En los últimos 10 años registró un crecimiento de un 4\%, la cifra más alta en comparación con el resto de los países latinoamericanos, y se estima que alcance un alto crecimiento, dadas las tendencias favorables, tanto a nivel nacional como internacional. Sólo en el 2014, se incorporaron 43,500 hectáreas, de las cuales 33,000 pertenecen a las empresas agroexportadoras, quienes propician la ampliación de la frontera agrícola en áreas de los grandes proyectos de irrigación y que repercuten favorablemente en las agroexportaciones peruanas sobre la tendencia mundial de consumo de alimentos naturales y orgánicos en el ámbito nacional e internacional (Miranda, 2017, p. 7).

El espárrago es una planta herbácea que pertenece a la familia de las Liliáceas y su nombre científico es Asparragus officinalis L. Asimismo, existen varios tipos, de los cuales los que se producen en el Perú son blancos y verdes. El espárrago blanco se emplea en mayor proporción para productos en conserva; mientras que el verde se exporta, principalmente, como fresco y congelado (Cuyuye, Planas, Salazar, 2017, p. 20).

El Perú es el segundo exportador mundial de espárrago verde con un 33\% del volumen total exportado en el 2016, superado por México con un 37\%, según Trademap. (Informática, 2017). En general, nuestro país tiene un lugar importante en cuanto a agro exportaciones de espárrago a nivel mundial, es así que: Cuyuche et. al (2008) sostienen que el Perú produce los mayores volúmenes de espárragos frescos a nivel mundial (ya que posee 26000 hectáreas cultivadas), posee el tercer mayor rendimiento productivo (12 000 kilogramos por hectárea); y además, es segundo en la producción de espárragos procesados. La costa peruana, a pesar de las condiciones adversas propias de la naturaleza, es el área geográfica en el cual se producen los mayores volúmenes de espárragos (Vázquez-Rowe, Kahhat, Quispe y Bentín, 2016, como se citó en Salas-Canales, 2020); además, históricamente, su cultivo empezó en la década de 1950 en la región de La Libertad, gracias a unas semillas traídas desde California y luego de 1980, la producción se incrementó debido a que comenzó a cultivarse en la región Ica (Schuster y Maertens, 2013, como se citó en Salas-Canales, 2020).

Según Koo (2020) el Perú exporta espárragos en tres presentaciones: frescos, conservas y congelados. Alrededor del $73 \%$ de las exportaciones corresponde a fresco, un $21 \%$ en conserva y un $6 \%$ a congelados. Durante el año 2016, el Perú se ubicó como el segundo país exportador de espárragos frescos del mundo con el 33\% del volumen total exportado superado por México con un 37\% (Gestión 2017). El año 2017, las exportaciones peruanas de espárragos frescos alcanzaron un valor de 388 millones de dólares siendo su principal mercado de destino Estados Unidos (como se citó en Cedano C., 2019).

Como lo menciona (Agap, 2019, citado en Cedano, 2019) las exportaciones peruanas de espárragos (frescos, en conserva y congelado) ascendieron a 169,807 toneladas, mostrando un crecimiento de un $15 \%$ respecto a las 147,708 toneladas despachadas el 2017. Durante el primer bimestre del presente año, el espárrago fue el principal producto exportado con 45 millones de dólares, y sus principales mercados 
fueron Estados Unidos con una participación de un 49\%, Holanda (20\%), Reino Unido y España (11\%).

En la cosecha, se procede a cortar el follaje a la altura de la superficie del suelo, llamándose a esta operación “chapodo” (Sánchez Velásquez y Sánchez Vigo, 2008, p.45).

La cadena productiva del espárrago atraviesa una serie de etapas consecutivas que necesitan ser valuadas adecuadamente (ver Figura 1):

\section{Figura 1}

Cadena Productiva del Espárrago

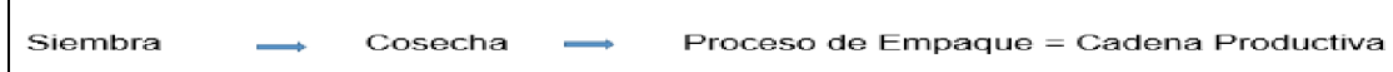

Cómo una propuesta al problema de la valorización adecuada del producto encontramos al sistema de costos por procesos "Los sistemas de costeo por procesos separan los costos en categorías de costos de acuerdo con el momento en que los costos se introducen al proceso" (Horngren, Datar, y Foster, 2007, p.609).

Los costos de una empresa surgen como base para tomar decisiones gerenciales, así como para la planeación y el control sobre la base de la información.

"La información de costos se utiliza también para la planeación y para el control.

Debería ayudar a los administradores a decidir qué, por qué, cómo debería hacerse

y qué tan bien se está haciendo. Por ejemplo, la información acerca de los ingresos

y de los costos para un nuevo producto podría utilizarse como un insumo para el costeo objetivo. En esta etapa, los ingresos y los costos esperados pueden cubrir la totalidad de la vida del nuevo producto. Por lo tanto, los costos de diseño proyectados, el desarrollo, las pruebas, la manufactura, la comercialización, la distribución y el servicio serían información esencial“" (Hansen, Mowen, 2007, p.31).

Según la investigación de Roldán, Agudelo y Hernández, (2013) citado en Cárdenas Arias, Guamán, Siguenza-Guzman y Segarra (2020) , se muestra que el sistema de costeo, por su parte, refleja los procedimientos que se realizan para la acumulación y el registro de costos, durante el proceso productivo..

Es importante destacar que, dadas las características del proceso de producción de una empresa, es necesario que se busque establecer el sistema que se deberá utilizar para la generación apropiada de la información de costos, pudiendo ser este sistema flexible y confiable además de proporcionar información para varios propósitos y responder a diferentes tipos de preguntas. En general, el sistema se utiliza para satisfacer las necesidades de acumulación, medición y asignaciones de costos. La acumulación de costos es el reconocimiento y el registro de los costos. La medición de costos implica la determinación de importes monetarios de los materiales directos, de la mano de obra directa y de los costos indirectos utilizados en producción. La asignación de costos es la asociación de los costos de producción con las unidades producidas (Hansen, Mowen, 2007, p.185).

Es también propio del sistema de costos por procesos, el que, una vez iniciado un proceso determinado, ya sea de una parte específica o del producto mismo, no se puede 
interrumpir porque es de naturaleza contínua, en donde los procesos se adelantan en forma secuencial, es decir, que el proceso adelantado en un departamento de producción requiere continuar en otro, y en otro, hasta salir finalmente terminado hacia el almacén, o en forma paralela, en donde el proceso en cada departamento es independiente el uno del otro, y al final se juntan o ensamblan todos los procesos para obtener el artículo deseado. (Velástegui, 2012).

Hansen y Mowen (2007) afirman respecto a los elementos que conforman el costo por proceso requiere de insumos materiales de mano de obra y de costos indirectos, por lo general en iguales cantidades para cada unidad de producto. (p.227).

La mano de obra directa descrita para Horgren et al. (2012) incluye las remuneraciones de toda la mano de obra de fabricación que se pueda identificar como objeto del costo en forma económicamente factible, como los sueldos y prestaciones pagadas.

En cuanto a los costos indirectos, Hansen y Mowen (2007) establecen que no se pueden rastrear fácilmente a los objetos de costo, es decir, que no existe relación causal entre el costo y el objeto del costo o que el rastreo no es económicamente factible, además menciona que la distribución de los costos indirectos entre los objetos de costo recibe el nombre de distribución. Toda vez que no existe relación causal, la asignación de costos indirectos se basa en los vínculos de conveniencia o afectación (p. 37). "Los costos indirectos son aquellos que no pueden ser rastreados en forma sencilla y exacta a un objeto de costo.” (Hansen y Mowen, 2007).

Se debe tomar en cuenta que el sistema de costeo por procesos es más sencillo

que un sistema de costos por órdenes, debido a que este último tiene más cuentas de producción. Además, en un sistema de costeo por procesos, no hay trabajos individuales, no se utilizan hojas de costo para las órdenes y no se necesita dar seguimiento a los materiales empleados hacia cada orden particular. Los materiales se rastrean en los procesos, pero existen mucho menos procesos que trabajos en un sistema de órdenes. Además, no hay necesidad de utilizar boletas de trabajo para asignar los costos de la mano de obra a los procesos. Ya que los empleados trabajan por lo general la totalidad de su turno dentro de un proceso en particular, no se necesita un seguimiento detallado de la mano de obra. De hecho, en muchas empresas, los costos de la mano de obra son un porcentaje tan pequeño de los costos del proceso total que simplemente se combinan con los costos indirectos, creando una categoría de costo de conversión (Hansen y Mowen, 2007, p.229).

Los costos acumulados en el proceso A se acumulan y pasan a formar parte del costo inicial del proceso B y estos a su vez acumulados forman parte del costo inicial del proceso C. (ver Figura 2)

\section{Figura 2}

Flujo del Costeo por Procesos

Costos Proceso A + Costos Proceso B + Costos Proceso C $=$ Costos Totales

Costos Totales $/$ Unidades producidas $=$ Costo Unitario 


\section{MATERIAL Y MÉTODOS}

Las variables de estudio son "costo por procesos" y "cadena productiva del espárrago", es decir se busca demostrar el impacto positivo de la aplicación del costeo por procesos sobre la cadena productiva del espárrago. El tipo de investigación fue descriptivo porque únicamente pretende medir o recoger información de manera independiente o conjunta sobre los conceptos o las variables a las que se refieren. El diseño fue descriptivo no experimental; esto debido a que la investigación describe situaciones que se presentan sin interferir en la realidad, observando los fenómenos tal y como se dan en su contexto natural para después analizarlos.

La muestra está conformada por las medianas empresas agro exportadoras ubicadas en la región de La Libertad centrándonos en el ejercicio 2018, y para nuestro análisis utilizaremos el estudio de caso, el cual será aplicado a la empresa Vivadis Perú S.A.C., empresa dedicada principalmente a la agroexportación de espárragos y que se encuentra ubicada en dicha región. Si bien la empresa opera desde el año 2012 y su principal cliente se encuentra en Canadá, el volumen de ventas no la incluye en la lista de principales agroexportadores de la región de La Libertad, sin embargo, la empresa fue escogida debido a la experiencia que tuvo el autor de laborar en esa institución.

A continuación, se muestran las principales medianas empresas agro exportadoras de espárragos según la Comisión para la Promoción de Exportaciones en el Perú (PROMPEX) (ver Tabla 1).

\section{Tabla 1}

Principales Medianas Empresas Exportadoras de Espárragos

\section{PRINCIPALES MEDIANAS EMPRESAS EXPORTADORAS DE ESPÁRRAGOS}

\begin{tabular}{|c|c|c|c|c|}
\hline $\mathrm{N}^{\circ}$ & EMPRESA & DIRECCIÓN & R.U.C. & $\begin{array}{l}\text { PARTICIPACIÓN } \\
\text { DE MERCADO* }\end{array}$ \\
\hline 1 & Camposol S.A. & $\begin{array}{l}\text { Calle Augusto Tamayo } \mathrm{N}^{\circ} 180 \\
\text { Lima - San Isidro }\end{array}$ & 20340584237 & 0.12 \\
\hline 2 & Sociedad Agrícola Virú & $\begin{array}{l}\text { Carretera Panamericana Norte } N^{\circ} \\
521 \text { - Virú - La Libertad }\end{array}$ & 20373860736 & 0.11 \\
\hline 3 & $\begin{array}{l}\text { Sociedad Agrícola } \\
\text { DROKASA S.A. }\end{array}$ & $\begin{array}{l}\text { Carretera Panamericana Sur } \\
\text { N³12 Fundo Santa Rita Ica - Ica - Santiago }\end{array}$ & 20325117835 & 0.09 \\
\hline 4 & $\begin{array}{l}\text { DANPER } \\
\text { TRUJILLO S.A.C. }\end{array}$ & $\begin{array}{l}\text { CARR. Industrial A Laredo No SN FND. } \\
\text { Barrio Nuevo La Libertad - } \\
\text { Trujillo - Moche }\end{array}$ & 20170040938 & 0.07 \\
\hline 5 & IQF DEL PERÚ & $\begin{array}{l}\text { Av. Alberto del Campo No } 405 \text { Res. } \\
\text { Orrantia Lima - Lima- San Isidro }\end{array}$ & 20100032709 & 0.05 \\
\hline 6 & $\begin{array}{l}\text { Complejo } \\
\text { Agroindustrial Beta S.A. }\end{array}$ & $\begin{array}{l}\text { Calle Luis Gálvez Ronceros Nº } \\
117 \text { Ica - Chincha - Chincha Alta } \\
\text { Carr.Panamericana Norte Km. }\end{array}$ & 20297939131 & 0.04 \\
\hline 7 & GREEN PERÚ S.A. & $\begin{array}{l}542 \text { Sector CHAVIMOCHIC La } \\
\text { Libertad - Trujillo }\end{array}$ & 20397680038 & 0.04 \\
\hline 8 & $\begin{array}{l}\text { AGRO INDUS- } \\
\text { TRIAS AIB S.A. }\end{array}$ & $\begin{array}{l}\text { Av. Ricardo Palma No } 894 \text { San } \\
\text { Antonio - Miraflores - Lima } \\
\text { Carretera Panamericana Sur No }\end{array}$ & 20104420282 & 0.04 \\
\hline 9 & Agrícola Athos S.A. & $\begin{array}{l}294 \\
\text { Fundo Los Pobres Ica - Ica Salas }\end{array}$ & 20367472694 & 0.03 \\
\hline
\end{tabular}


*La participación de mercado está en relación al total de exportaciones en millones de US\$ Fuente: PROMPEX

Elaboración: AGROBANCO

\section{RESULTADOS}

A través de la Tabla 2 se muestra la aplicación del sistema de costos por procesos en cada una de las etapas de la cadena productiva del espárrago. Las etapas para nuestro estudio se han dividido en: siembra, cosecha y proceso de empaque y a su vez, cada etapa se ha subdividido de acuerdo a las actividades que las conforman, tal como se visualiza en la columna denominada "detalle".

Las actividades que forman parte de cada etapa requieren de insumos y de mano de obra, en la Tabla 2 se muestran éstas tomando en cuenta las unidades de medida que les corresponde, ya sea horas máquina (Hr/máquina), kilogramos (Kg.), horas hombre ( $\mathrm{Hr} /$ hombre), metros cúbicos (m3) y unidades (unid.).

Así mismo en la Tabla 2 se muestran las cantidades de insumos utilizados en cada actividad de acuerdo con la necesidad de la producción, es así por ejemplo en la etapa de siembra encontramos las actividades de: preparación de terreno, donde se utiliza el arador, el gradeo, la nivelación del terreno y el proceso de surcado, que son maquinarias utilizadas en esa etapa.

La mano de obra es aplicada de acuerdo con un control de tiempos que se establece en la empresa a través de partes diarios, esto con el fin de aplicar el costo por horas empleadas en cada proceso productivo.

\section{Tabla 2.}

Costos por Procesos Aplicados al Caso Vivadis Perú S.A.C.

Expresados en Nuevos Soles (S/) Moneda Peruana

VIVADIS PERÚ S.A.C.

AÑO: 2018

PRODUCTO: ESPÁRRAGO VERDE FRESCO 5.23 Has de almacigo

\begin{tabular}{|c|c|c|c|c|c|c|}
\hline \multirow[t]{3}{*}{ DETALLE } & \multicolumn{6}{|c|}{ REQUERIMIENTO POR HECTÁREAS (Hás) } \\
\hline & \multirow[t]{2}{*}{ UNID. } & \multirow{2}{*}{$\begin{array}{l}\text { CANT. } \\
\text {. / Hás }\end{array}$} & \multirow{2}{*}{$\begin{array}{l}\text { COSTO } \\
\text { UNIT. } \\
\text { s/ }\end{array}$} & \multirow{2}{*}{$\begin{array}{l}\text { COSTO } \\
\text { HR/MAQ } \\
\text {. / Hás }\end{array}$} & \multicolumn{2}{|l|}{ TOTAL } \\
\hline & & & & & $\mathrm{S} /$ & $\%$ \\
\hline SIEMBRA & & & & & 48584,62 & \\
\hline 1. PREPARACIÓN & & & & & 2565,23 & 100,00 \\
\hline INSUMOS & & & & & 1995,94 & 77,81 \\
\hline Arador & Hr/Máquina & 2 & 29,508 & 59,016 & 308,66 & 12,03 \\
\hline Gradeo & Hr/Máquina & 2 & 13,115 & 26,230 & 137,18 & 5,35 \\
\hline Nivelación & Hr/Máquina & 2 & 26,230 & 52,459 & 274,36 & 10,70 \\
\hline Proceso de surcado & Hr/Máquina & 2 & 13,115 & 26,230 & 137,18 & 5,35 \\
\hline MANO DE OBRA & & & & & 569,28 & 22,19 \\
\hline
\end{tabular}




\begin{tabular}{|c|c|c|c|c|c|c|}
\hline Mano de obra & $\mathrm{Hr} /$ Hombre & 24 & 4,535 & 108,850 & 569,28 & 22,19 \\
\hline 2. RIEGO Y SEMILLA & & & & & 44544,72 & 100,00 \\
\hline INSUMOS & & & & & 43690,80 & 98,08 \\
\hline Abono de ganado & $\mathrm{Kg}$. & 20000 & 0,017 & 332,498 & 1738,96 & 3,90 \\
\hline Riego post abonado & M3 & 2000 & 0,026 & 51,312 & 268,36 & 0,60 \\
\hline Riego pasado de mula & M3 & 850 & 0,026 & 21,807 & 114,05 & 0,26 \\
\hline Humus & $\mathrm{Kg}$. & 5000 & 0,023 & 115,451 & 603,81 & 1,36 \\
\hline Urea & $\mathrm{Kg}$. & 456 & 0,472 & 215,293 & 1125,98 & 2,53 \\
\hline Fosfato diamónico & $\mathrm{Kg}$. & 152 & 0,603 & 91,698 & 479,58 & 1,08 \\
\hline Cloruro de Potasio & $\mathrm{Kg}$. & 200 & 0,420 & 83,935 & 438,98 & 0,99 \\
\hline Ácido fosfórico & $\mathrm{Kg}$. & 140 & 0,944 & 132,178 & 691,29 & 1,55 \\
\hline \multirow[t]{2}{*}{ Semilla } & $\mathrm{Kg}$. & 8 & 913,714 & $7,309,709$ & 38229,78 & 85,82 \\
\hline & & & & & 853,93 & \\
\hline Abonado de ganado & Hr/Hombre & 6 & 4,535 & 27,212 & 142,32 & 0,32 \\
\hline Pasada de mula & Hr/Hombre & 6 & 4,535 & 27,212 & 142,32 & 0,32 \\
\hline Marcado & $\mathrm{Hr} /$ Hombre & 6 & 4,535 & 27,212 & 142,32 & 0,32 \\
\hline Colocación de semilla & Hr/Hombre & 18 & 4,535 & 81,637 & 426,96 & 0,96 \\
\hline 3. CULTIVO & & & & & $1,474,67$ & 100,00 \\
\hline INSUMOS & & & & & 270,22 & 18,32 \\
\hline Fetrilon Combi & $\mathrm{Kg}$. & 1.5 & 22,951 & 34,426 & 180,05 & 12,21 \\
\hline This Micromix & $\mathrm{Kg}$. & 1.5 & 11,494 & 17,241 & 90,17 & 6,11 \\
\hline MANO DE OBRA & & & & & $1,204,45$ & 81,68 \\
\hline Control Fitosanitario & $\mathrm{Hr} /$ Hombre & 6 & 4,535 & 27,212 & 142,32 & 9,65 \\
\hline Deshierbo & Hr/Hombre & 6 & 4,535 & 27,212 & 142,32 & 9,65 \\
\hline Riego & M3 & 6855 & 0,026 & 175.871 & 919,80 & 62,37 \\
\hline COSECHA & & & & & 6090,53 & \\
\hline 1. RECOLECCIÓN & & & & & 16,28 & 100,00 \\
\hline INSUMOS & & & & & 231,64 & 44,87 \\
\hline Homai & $\mathrm{Kg}$. & 0.5 & 31,805 & 15,902 & 83,17 & 16,11 \\
\hline Orthene & $\mathrm{Kg}$. & 0.5 & 34,481 & 17,241 & 90,17 & 17,47 \\
\hline Dipterex & $\mathrm{Kg}$. & 0.5 & 22,294 & 11,147 & 58,30 & 11,29 \\
\hline MANO DE OBRA & & & & & 284,64 & 55,13 \\
\hline Rastrillada & $\mathrm{Hr} /$ Hombre & 1 & 4,535 & 23,72 & 23,72 & 4,59 \\
\hline Riego pre cosecha & $\mathrm{Hr} /$ Hombre & 1 & 4,535 & 23,72 & 23,72 & 4,59 \\
\hline Chapodo y limpieza & $\mathrm{Hr} /$ Hombre & 8 & 4,535 & 189,76 & 189,76 & 36,76 \\
\hline Guardianía en cosecha & $\mathrm{Hr} /$ Hombre & 2 & 4,535 & 47,44 & 47,44 & 9,19 \\
\hline 2. SELECCIÓN & & & & & 5574,25 & 100,00 \\
\hline MANO DE OBRA & & & & & 5574,25 & 100,00 \\
\hline Clasificación & Hr/Hombre & 150 & 4,535 & 680,31 & 3558,03 & 63,83 \\
\hline Pre lavado & $\mathrm{Hr} /$ Hombre & 85 & 4,535 & 385,51 & 2016,22 & 36,17 \\
\hline
\end{tabular}




\begin{tabular}{|c|c|c|c|c|c|c|}
\hline \multirow{2}{*}{$\begin{array}{l}\text { PROCESO DE EMPAQUE } \\
\text { 1. PESAJE DEL ATADO }\end{array}$} & & & & & \multicolumn{2}{|l|}{5644,41} \\
\hline & & & & & 1267,35 & 100,00 \\
\hline INSUMOS & & & & & 954,41 & 75,31 \\
\hline Herramientas y accesorios & Und. & 132 & 2,330 & 307,560 & 307,56 & 24,27 \\
\hline Balanza electrónica & Und. & 5 & 124,510 & 622,550 & 622,55 & 49,12 \\
\hline Canastas recolectoras & Und. & 100 & 0,243 & 24,300 & 24,30 & 1,92 \\
\hline MANO DE OBRA & & & & & 12,94 & 24,69 \\
\hline Traslado de productos & Hr/Hombre & 2 & 4,535 & 9,070 & 9,07 & 0,72 \\
\hline Corte de atados & Hr/Hombre & 43 & 4,535 & 195,022 & 195,02 & 15,39 \\
\hline Control calidad del producto & Hr/Hombre & 24 & 4,535 & 108,850 & 108,85 & 8,59 \\
\hline 2. EMBALAJE & & & & & 4377,06 & 100,00 \\
\hline INSUMOS & & & & & 4256,35 & 97,24 \\
\hline Ligas, etiquetas, zunchos & Und. & 3835 & 0,950 & 3643,25 & 3643,25 & 83,24 \\
\hline Cajas plásticas & Und. & 320 & 1,570 & 502,40 & 502,40 & 11,48 \\
\hline Parihuelas & Und. & 54 & 2,050 & 110,70 & 110,70 & 2,53 \\
\hline MANO DE OBRA & & & & & 120,71 & 2,76 \\
\hline Manipuleo & Hr/Hombre & 15 & 4,535 & 68,031 & 68,03 & 1,55 \\
\hline Almacenaje & $\mathrm{Hr} /$ Hombre & 5 & 4,535 & 22,677 & 22,68 & 0,52 \\
\hline Transporte & $\mathrm{Hr} /$ Hombre & 2 & 15,000 & 30,000 & 30,00 & 0,69 \\
\hline SUB TOTAL & & & & & 60319,56 & \\
\hline
\end{tabular}

Observamos en la Tabla 2 en la etapa de siembra, que los insumos utilizados en la preparación del terreno representan el 77,81\% mientras que la mano de obra utilizada es el $22,19 \%$, esto se debe a que en esta etapa predomina el uso de la maquinaria necesaria para preparar el terreno y llevar a cabo la siembra.

Posteriormente tenemos el riego donde los insumos representan el 98,08\% del valor de este, esto se debe a que en esta actividad se busca que el terreno se encuentre cubierto en sus necesidades hídricas y de abono que le proporcionará los nutrientes para el crecimiento de las plántulas.

El cultivo representa la última actividad de la siembra, aquí podemos observar que los insumos representan el 18,32\% del total del cultivo, siendo el producto predominante el Fetrilon Combi que es un fertilizante que va a aportar múltiples nutrientes al producto, además del apoyo de la mano de obra en el control fitosanitario, el deshierbe y el riego.

En la siguiente etapa denominada "cosecha" se procede a recoger los productos luego de haber superado el proceso de maduración, encontrando aquí dos actividades muy importantes como son: la recolección y la selección. La recolección se produce cuando los espárragos han logrado cierto grado de crecimiento y necesitan ser separadas de la tierra, siendo la utilización de insumos en esta actividad del 44,87\% del total de la recolección, mientras que la mano de obra representa el 55,13\%. Dentro de la mano de obra que se utiliza en la recolección encontramos actividades tales como la rastrillada del terreno, el riego precosecha, el chapodo y limpieza del suelo. 
Posteriormente tenemos como última actividad dentro de la etapa de cosecha a la selección, la misma que consiste en clasificar los espárragos de acuerdo con los tamaños o grosores de este, esto debido a las preferencias de los mercados a los cuales se destinará el producto. El proceso de clasificación dentro de la actividad de selección representa el $63,83 \%$ del total de la mano de obra, mientras que la diferencia corresponde al proceso de prelavado del producto, el cual es necesario para poder ingresar a la siguiente etapa del proceso productivo.

Finalmente, encontramos la última etapa denominada "proceso de empaque", la cual consiste en proteger el producto y conservarlo para su próximo traslado. Durante esta etapa contamos con el pesaje del atado y el embalaje de este. En cuanto al pesaje del atado, los insumos utilizados en esta actividad representan el 75,31\% del total, mientras que la mano requerida representa el $24,69 \%$.

Para el pesaje del atado se utilizan herramientas y accesorios, así como balanzas electrónicas para controlar el peso del atado y canastas recolectoras, las cuales permitirán la acumulación del producto en condiciones adecuadas. En esta etapa, suele ser importante la aplicación de la mano de obra para llevar adelante los procesos antes descritos principalmente en el control de calidad del producto.

Mediante el embalaje, el producto estará listo para ser entregado a su destino. En este proceso se cuenta con insumos tales como las ligas, etiquetas, zunchos, cajas plásticas y parihuelas, representando estos productos el $97,24 \%$ del total del embalaje, mientras que la mano de obra para el manipuleo, el almacenaje y el transporte representan el 2,76\% de la actividad.

Posteriormente, mostramos en la Tabla 3 denominada "costeo empírico aplicado al caso. Vivadis Perú S.A.C." La aplicación de un sistema de costeo donde los cálculos se realizan en base a la experiencia en la labor, es decir, no se separa de manera sistemática los costos de acuerdo con la aplicación más exacta de sus elementos. La asignación de la mano de obra se realiza en base a estándares conocidos en las horas que se utilizan en cada labor y la aplicación de la materia prima se realiza en base a porcentajes tomando en cuenta el conocimiento en la práctica de los insumos utilizados en cada proceso productivo.

En esta tabla mostramos la etapa de siembra, donde los insumos utilizados representan el 79,74\% del total, mientras que la mano de obra representa el 20,26\%. Cabe mencionar que la mano de obra utilizada en esta etapa es muy importante para lograr que el suelo, donde se llevará a cabo la siembra, se encuentre en óptimas condiciones y ofrezca al producto los nutrientes necesarios para su normal desenvolvimiento.

Así mismo podemos observar que en el proceso de riego y semilla, los insumos utilizados en la producción representan el $98,14 \%$ de la actividad mientras que la mano de obra requerida es aproximadamente el 1,86\%, cabe indicar que el valor más importante dentro de este proceso es el valor de la semilla.

Dentro de lo que representa el cultivo, en este proceso la mano de obra equivale al $82,25 \%$ del total mientras que los insumos que se utilizan en este proceso tienen un porcentaje del 17,75\% del total del cultivo. 
En la etapa de cosecha, observamos también los procesos de recolección y selección, los cuales también son muy importantes en nuestra cadena productiva. En la recolección vemos que los insumos utilizados representan el 44,57\% mientras que la mano de obra equivale al 55,43\% del total del proceso. En la selección de espárragos el requerimiento de mano de obra abarca el 100\% del proceso.

En la etapa de empaque tenemos el pesaje del producto, en el cual los insumos utilizados representan el 72,96\% del total mientras que la mano de obra es el 27,04\% del total del proceso.

Finalmente, tenemos el embalaje, donde el consumo de insumos equivale al 96.41\% mientras que la mano de obra representa el 3,59\%.

\section{Tabla 3}

Costeo Empirico Aplicado al Caso Vivadis Perú S.A.C.

Expresados en Nuevos Soles (S/) Moneda Peruana

\section{VIVADIS PERÚ S.A.C.}

\begin{tabular}{|c|c|c|}
\hline $\begin{array}{l}\text { AÑO } \\
\text { PRODUCTO }\end{array}$ & $\begin{array}{l}2018 \\
\text { ESPÁRRAGC }\end{array}$ & \\
\hline$\overline{\text { ETAPA }}$ & MONTO S/ & $\%$ \\
\hline 1. SIEMBRA & $59,558,70$ & \\
\hline PREPARACIÓN & $3,278,55$ & 100,00 \\
\hline INSUMOS-PROCESO & $2,614,39$ & 79,74 \\
\hline MANO DE OBRA & 664,16 & 20,26 \\
\hline 2. RIEGO Y SEMILLA & $54,758,04$ & 100,00 \\
\hline INSUMOS & $53,738,07$ & 98,14 \\
\hline MANO DE OBRA & $1,019,97$ & 1,86 \\
\hline 3. CULTIVO & $1,522,11$ & 100,00 \\
\hline INSUMOS & 270,22 & 17,75 \\
\hline MANO DE OBRA & $1,251,89$ & 82,25 \\
\hline COSECHA & 6776,18 & \\
\hline 1. RECOLECCIÓN & 727,53 & 100,00 \\
\hline INSUMOS & 324,29 & 44,57 \\
\hline MANO DE OBRA & 403,24 & 55,43 \\
\hline 2. SELECCIÓN & 6048,65 & 100,00 \\
\hline INSUMOS & 0,00 & 0,00 \\
\hline MANO DE OBRA & 6048,65 & 100,00 \\
\hline EMPAQUE & 5722,91 & \\
\hline 1. PESAJE DEL ATADO & 1308,17 & 100,00 \\
\hline INSUMOS & 954,41 & 72,96 \\
\hline MANO DE OBRA & 353,76 & 27,04 \\
\hline 2. EMBALAJE & 4414,74 & 100,00 \\
\hline
\end{tabular}




\begin{tabular}{lll} 
INSUMOS & 4256,35 & 96,41 \\
MANO DE OBRA & 158,39 & 3,59 \\
\hline SUB TOTAL & $72,057,79$ & \\
\hline
\end{tabular}

Observando la Tabla 3 visualizamos que, utilizando el sistema de costos por procesos en la preparación para la siembra, los insumos representan el 77.81\% y la mano de obra equivale al $22.19 \%$ mientras que aplicando el costeo aplicado en base a la experiencia este representa el $79.74 \%$ y el $20.26 \%$ respectivamente. Esto se debe a que utilizando el sistema de costos por procesos el consumo de cada insumo utilizado es aplicado en base a la necesidad del mismo, de la misma manera en cuanto a la mano de obra, se discrimina en cada etapa las horas efectivamente trabajadas y se van asignando según el tiempo dedicado a cada actividad.

En cuanto a la etapa de cultivo, podemos observar que los insumos que se utilizan representan el $18.32 \%$ y la mano de obra el $81.68 \%$ según el costeo por procesos, mientras que utilizando el costeo en base a la experiencia estos representan el $17.75 \%$ y $82.25 \%$. Podemos observar que la mano de obra representa la mayor parte del costo en la etapa ya sea aplicando el costeo por procesos o el costeo en base a la experiencia, por lo tanto, es muy importante acumular adecuadamente las horas en esta labor y llevar un control concienzudo, de tal manera que se refleje confiablemente el costo por mano de obra.

En el proceso de recolección, aplicando el sistema de costeo por procesos, los insumos aplicados representan el $44.87 \%$ y la mano de obra $55.13 \%$ mientras que en el costeo aplicado en base a la experiencia representan el $44.57 \%$ y $55.43 \%$ respectivamente, en este caso si bien la diferencia puede no ser material, sin embargo, aplicado a cantidades importantes de insumos podría ocasionar distorsión en los costos. Es importante conocer la necesidad exacta de los insumos aplicados en este proceso tal como nos lo brinda el sistema de costeo por procesos.

En cuanto al proceso de selección, aplicando el sistema de costos por procesos el valor de la mano de obra se encuentra más alto que aplicando el costo en base a la experiencia, para esto es importante que se lleve el control adecuado de las horas empleadas en la labor de seleccionar los espárragos.

Finalmente tenemos la etapa de empaque, donde el pesaje del atado de espárragos tiene como insumos utilizados el $75.31 \%$ y la mano de obra $24.69 \%$ del total del proceso, mientras que con el costeo en base a la experiencia representan el $72.96 \%$ y el $27.04 \%$, lo cual nos permite deducir que las herramientas utilizadas tomando el costeo por procesos es mucho más precisa debido a que muestra con mayor exactitud la cantidad de insumos y la cantidad de horas que se utilizan para pesar cada atado del espárrago.

Así mismo, en el embalaje, los insumos representan el $97.24 \%$ y la mano de obra $2.76 \%$ en el costeo por procesos mientras que $96.41 \%$ y $3.59 \%$. Como en el caso del cultivo, en estas etapas predomina la mano de obra, por lo tanto, es muy importante el control de horas y la correcta acumulación de estas como lo ofrece el sistema de costos por procesos.

Podemos observar entonces que la acumulación de costos ya sea por materia prima como por mano de obra es muy importante en cada etapa del proceso productivo y 
presentar los datos con mayor precisión y de manera ordenada es propio del sistema de costos por procesos, presentándose entonces este sistema de costeo como una alternativa favorable para determinar los costos en la cadena productiva del espárrago.

\section{DISCUSIÓN}

Siendo el objetivo demostrar el impacto positivo de los costos por procesos en la valuación de la cadena productiva del espárrago y observando los resultados obtenidos en las tablas 2 y 3 , podemos deducir que la necesidad de información de los costos de materia prima y mano de obra se muestran más detallados y precisos con la aplicación del costeo por procesos, lo que permitiría a la gerencia tomar mejores decisiones. Los sistemas de costos deben de ser aplicados según la necesidad de la empresa, esto concuerda con Sánchez (2009) quien sostiene que luego de haber revisado los diversos conceptos y clasificaciones de costos, se puede afirmar que no existe una sola forma de clasificar los costos que responda a los requerimientos de costeo de toda empresa y que la clasificación de costos que debe elegir una compañía depende del giro de negocio y del objeto de costo (bienes tangibles e intangibles) que brinde y que también para estudiar y comprender los diversos términos de costos permite a los usuarios de la información de costos a tomar mejores decisiones de inversión, es decir que se debe adecuar la información a las necesidades de la empresa.

Tomar decisiones en base a costos elevados de materia prima o insumos puede conducir a la gerencia a buscar proveedores que ofrezcan mejores precios, esto con el fin de enfrentar a la competencia cada vez más agresiva, tomando en cuenta que en el mercado se encuentran empresas con procesos cada vez más mecanizados lo cual les permite reducir costos. Así mismo la empresa también puede caer en la tentación de buscar productos sustitutos, los cuales muchas veces suelen no ofrecer la condición requerida para lograr con éxito la producción del espárrago.

Por otro lado, se podría afectar la calidad del producto si la gerencia en base a la información de costos y con el fin de ser competitivos, toma la decisión de mejorar los costos reduciendo las cantidades de insumos que corresponden aplicar a la producción. La importancia del consumo de espárrago radica en su nivel nutricional, por lo que las cantidades de insumos a utilizar garantizan la calidad de este.

En cuanto a la mano de obra, el costeo inadecuado del mismo podría generar que la cantidad de horas dedicadas a la producción no se ajuste a la realidad, lo que haría que el costo del producto a este nivel se presente por debajo de lo real o se presente en sobre costo. Si se presenta por debajo de su valor real puede ocasionar que la empresa ofrezca precios con márgenes inferiores a lo que se espera lograr, caso contrario puede generar que los precios no sean competitivos.

Como consecuencia, para valuar adecuadamente los costos de producción de la cadena productiva de espárrago se presenta como una alternativa favorable el sistema de costos por procesos ya que nos permite conocer no sólo los costos acumulados por etapas, sino que contribuye confiablemente con la necesidad de información para la toma de decisiones. 


\section{ORCID}

Nelly Adriana Risco: Mc Gregor, Universidad Nacional Mayor de San Marcos, Lima, Perú

\section{FUENTE DE FINANCIAMIENTO}

El estudio fue autofinanciado.

\section{CONFLICTOS DE INTERÉS}

$\mathrm{El}$ autor declara que no existe conflicto de intereses.

\section{AGRADECIMIENTO}

No aplica.

\section{PROCESO DE REVISIÓN}

Este estudio ha sido revisado por pares externos en modalidad de doble ciego.

\section{DECLARACIÓN DE DISPONIBILIDAD DE DATOS}

No aplica.

\section{REFERENCIAS BIBLIOGRÁFICAS}

Agrobanco. Cultivo del Espárrago. Recuperado de: https://bit.ly/3xhbaFo

Cárdenas Arias, B.; Guamán, M.; Siguenza-Guzman, L.; y, Segarra, L. (2020). Integración de información de costos para la toma de decisiones en industrias de ensamblaje. Revista Economía y Política. Número 31. doi: 10.25097/rep.n31.2020.07

Castillo Bazalar, J. R. (1983). Contabilidad Agrícola. Lima, Perú: Ital Perú S.A.C.

Cuyuche L.; Planas, J.; Salazar, F. (2017). Planeamiento Estratégico del Espárrago en el Perú. [Tesis de maestría]. Pontificia Universidad Católica del Perú. Recuperado de: https://bit.ly/2Qneaj9

Delgado De La Flor, F., Montauban Del Solar, R., \& Hurtado, F. (1993). Cultivo del Espárrago.

Lima, Perú: Editorial Proyecto TIA Actividad 3B.

Dirección General de Promoción Agraria (2015). Las Cadenas Productivas. Recuperado de: https://bit.ly/3eznBnD

Exportación peruana de espárragos caería en el 2017 y se recuperaría en el 2018 (2017, 3 de octubre). Diario Gestión. Recuperado de: https://bit.ly/3tTnAkC

Hansen, D. R., y Mowen, M. M. (2003). Administración de Costos. México: International Thomson Editores, S.A. Recuperado de: https://bit.ly/2QtMWqQ

Hansen, D. y Mowen, M. (2007). Administración de Costos - Contabilidad y Control. México: Cengage Learning Editores, S.A.

Horngren, C.; Datar, S.; y, Foster, G. (2007). Contabilidad de Costos. México: Pearson. https://bit.ly/3vnZ8sd 
Koo, W. (2020, 28 de enero). Espárrago Fresco Perú Exportación 2019 diciembre. Agrodata Perú. Recuperado de: https://bit.ly/3eucPyL

Menores rendimientos afectarían a las exportaciones de espárragos este año (2019, 7 de abril). Diario Gestión. Recuperado de: https://bit.ly/3tXgKeg

Miranda, J. (2017). Importancia de la marca país para aprovechar las ventajas competitivas en las exportaciones de productos agrarios no tradicionales. [Tesis de maestría]. Universidad Ricardo Palma. Recuperado de: https://bit.ly/3esZEyh

O’Brien, Tim M. y Díaz Rodríguez, Alejandra (2004). Exportaciones agrícolas por medio del desarrollo y aplicación de normas de inocuidad y calidad. El ejemplo del espárrago peruano. Reporte del Programa de Sanidad Agropecuaria e Inocuidad de Alimentos del Instituto Interamericano de Cooperación para la Agricultura (IICA). Recuperado de: https://bit.ly/3aDEVXb

Polimeni, R., Fabozzi, F., \& Adelberg, A. (1994). Contabilidad de Costos. México: McGraw-Hill.

Producción nacional aumentó 4,81\% en enero de 2017 (2017, 15 de marzo). Instituto Nacional de

Estadística e Informática. Recuperado de: https://bit.ly/2S5g20j

Productores de Ica conquistan 105 países en todo el mundo (2017, 24 de septiembre). Diario Gestión. Recuperado de: https://bit.ly/3vk0Cnm

Rodríguez, A. (2004). Mejorando la competitividad y el acceso a los mercados de exportaciones agrícolas por medio del desarrollo y aplicación de normas de inocuidad y calidad. El ejemplo del espárrago Peruano. Reporte del Programa de Sanidad Agropecuaria e Inocuidad de Alimentos del Instituto Interamericano de Cooperación para la Agricultura (IICA). Recuperado de: https://bit.ly/3aDEVXb

Salas-Canales, H. L. (2020), Agroexportaciones no tradicionales y su contribución al desarrollo económico Peruano. Revista Científica Dominio de las Ciencias, Volumen 6. pp. 4-27. doi: http://dx.doi.org/10.23857/dc.v6i1.1343.

Sánchez Velásquez, G. y Sánchez Vigo, J. (2008). Manejo Integrado del Cultivo del Espárrago en el Perú. Instituto Peruano del Espárrago y Hortalizas. Recuperado de: https://bit.ly/2QyxIB5

Velástegui, W. (2016). Costos II. Metro Carolina Sur. Ecuador. Recuperado de: https://bit.ly/3vnZ8sd

Vázquez-Rowe, I., Kahhat, R., Quispe, I. y Bentín, M. (2016). Environmental profile of green asparagus production in a hyper-arid zone in coastal Peru. Journal of Cleaner Production, 112(4), 2505-2517. doi: 10.1016/j.jclepro.2015.09.076 